\title{
BOUNDS AND EXTREMAL CONFIGURATIONS FOR THE TORSIONAL RIGIDITY OF COATED FIBER REINFORCED SHAFTS
}

\author{
ROBERT LIPTON* AND TUNGYANG $\mathrm{CHEN}^{\dagger}$
}

\begin{abstract}
In this paper we derive bounds on the torsional rigidity for coated fiber reinforced shafts. The bounds are used to assess the optimality or suboptimality of fiber reinforcement configurations. This investigation focuses on coated fiber reinforcements with circular cross section. It is shown how the effective antiplane shear modulus and torsional rigidity of each coated fiber are used to determine whether the configuration provides reinforcement above or below that of a homogeneous shaft containing no coated fibers. Simply connected shaft cross sections of arbitrary shape reinforced with any configuration of coated fibers are considered. Precise conditions on the effective antiplane shear modulus and torsional rigidity of each coated fiber are given under which the circular shaft reinforced with a single centered circular coated fiber is either optimal or suboptimal.
\end{abstract}

Key words. Torsion, Coated Fibers

AMS subject classifications. 35J20,74Q99

1. Introduction. The problem of extremizing the torsional rigidity of prismatic shafts has been the focus of many investigations. For homogeneous shafts made from elastically isotropic material Saint-Venant (1856) proposed that among all prismatic shafts with given cross-sectional area that the greatest torsional rigidity is obtained by a shaft with circular cross section. This proposition was proven by Polya (1948). For multiply connected cross sections of given cross-sectional area Polya and Weinstein (1950) showed that the optimal cross section is given by the annulus. Alvino and Trombetti (1985) considered composite shaft cross sections made up of perfectly bonded elastic materials. Here each phase is a cylindrical fiber of arbitrary cross section with generators parallel to the shaft. In this context they showed that circular cross sections with a radially nonincreasing arrangement of compliance delivers the maximum torsional rigidity among all cross sections with given cross-sectional area and fixed area fraction of the constituent phases.

When the materials are imperfectly bonded the elastic displacement may suffer jumps across the interface between different elastic phases. To first order one models the imperfect bonding in terms of a linear constitutive law relating tangential stress to the jump in the warping displacement. This model for imperfect bonding is well known and is referred to as the spring layer model, see Jones and Whitter (1967). In this context one considers shafts reinforced with fibers of greater shear stiffness than the matrix. One is interested in extremizing the torsional rigidity over fiber configurations and understanding how the imperfect interface compromises the benefits of the stiffer reinforcement. It is found that the degree of imperfect bonding relative to the contrast in compliance between matrix and fiber explicitly determines the type of fiber configuration that maximizes the torsional rigidity, see Lipton (1998, Theorems 1.1 through 1.7). The relative degree of imperfect bonding is given by the parameter

$$
\mathrm{R}_{c r}=\frac{\alpha^{-1}}{\mathrm{G}_{m}^{-1}-\mathrm{G}_{f}^{-1}}
$$

\footnotetext{
*Department of Mathematics, Louisiana State University, Baton Rouge, LA 70803, U.S.A., (lipton@math.lsu.edu).

$\dagger$ Department of Civil Engineering, National Cheng Kung University Tainan 70101, Taiwan, (tchen@mail.ncku.edu.tw).To Appear in SIAM J. on Applied Mathematics 2004.
} 
where $\mathrm{G}_{f}$ is the shear modulus of the fiber reinforcement, $\mathrm{G}_{m}$ is the shear modulus of the matrix and $\alpha$ is the interfacial shear stiffness having dimensions of shear stiffness per unit length. For a shaft with circular cross section of radius $R$ containing $N$ reinforcement fibers of circular cross section with common radii given by $\mathrm{R}_{c r}$ the imperfect interface balances the reinforcing effect of the fibers and the warping function out side the fibers is precisely zero. For this case the torsional rigidity is independent of the location of the fibers and is given by

$$
\frac{\pi \mathrm{G}_{\mathrm{m}}}{2}\left(R^{4}-N \mathrm{R}_{c r}^{4}\right)+\frac{\pi \mathrm{G}_{\mathrm{f}}}{2} N \mathrm{R}_{c r}^{4}
$$

and is precisely the torsional rigidity of a circular shaft of radius $R$ reinforced with a single centered fiber of radius $N^{1 / 4} \mathrm{R}_{c r}$, see Lipton (1998).

In many composites a third phase or inter-phase separating fiber and matrix is present. The inter-phase or coating phase often has elastic properties that are distinct from the fiber or matrix. In this context the recent work of Chen, Benveniste and Chuang (2002) treats a system of $N$ fibers with circular cross section and radii $a_{i}$, $i=1, \ldots, N$. The fibers are coated by a shell of uniform thickness and the outer radius of the coated fiber is $b_{i}, i=1, \ldots, N$. The shear modulus if the $i^{\text {th }}$ fiber is denoted by $\mathrm{G}_{f}^{i}$ and the shear modulus of the associated coating is denoted by $\mathrm{G}_{c}^{i}$. The area fraction of the fiber phase in the $i^{\text {th }}$ coated fiber system is denoted by $\nu_{i}$ and $\nu_{i}=a_{i}^{2} / b_{i}^{2}$. One recalls the formula for the effective anti-plane shear modulus for the concentric coated cylinders assemblage of Hashin and Rosen (1964) given by

$$
G_{C C A}^{i}=\mathrm{G}_{c}^{i}\left(\frac{\mathrm{G}_{c}^{i}\left(1-\nu_{i}\right)+\mathrm{G}_{f}^{i}\left(1+\nu_{i}\right)}{\mathrm{G}_{c}^{i}\left(1+\nu_{i}\right)+\mathrm{G}_{f}^{i}\left(1-\nu_{i}\right)}\right) .
$$

Here $G_{C C A}^{i}$ gives the effective shear stiffness of each coated fiber. Chen, Benveniste and Chuang (2002) show that when the effective shear stiffness of each coated fiber equals the matrix shear stiffness $\mathrm{G}_{m}$, i.e.,

$$
G_{C C A}^{i}=\mathrm{G}_{m}, \quad i=1, \ldots, N,
$$

then the warping function outside the coated fibers is zero and the torsional rigidity is given by

$$
\mathcal{A}^{N}=\frac{\pi}{2} \mathrm{G}_{m} R^{4}+\sum_{i=1}^{N}\left(\frac{\pi}{2}\left(\mathrm{G}_{c}^{i}\left(b_{i}^{4}-a_{i}^{4}\right)+\mathrm{G}_{f}^{i} a_{i}^{4}\right)-\frac{\pi}{2} \mathrm{G}_{m} b_{i}^{4}\right) .
$$

When all fibers have the same radius and coating thickness $\ell$ one passes to the distinguished limit given by

$$
\lim _{\ell \rightarrow 0} \lim _{\mathrm{G}_{c}^{i} \rightarrow 0} \frac{\ell}{\mathrm{G}_{c}^{i}}=\alpha^{-1}
$$

in (1.4) and (1.5) to see that $\mathcal{A}^{N}$ is given by (1.2).

The relations given by (1.4) express the balance between the shear moduli of the matrix, fiber, coating and coating thickness that renders the warping function zero outside the inclusions. Furthermore under the hypotheses leading to (1.5) it is evident that if the torsional rigidity of each coated fiber given by

$$
T_{f}^{i}=\frac{\pi}{2}\left(\mathrm{G}_{c}^{i}\left(b_{i}^{4}-a_{i}^{4}\right)+\mathrm{G}_{f}^{i} a_{i}^{4}\right)
$$


equals the torsional rigidity $\frac{\pi}{2} \mathrm{G}_{m} b_{i}^{4}$, obtained by replacing coating and fiber shear moduli with the matrix shear moduli, then there is complete neutrality, i.e., the torsional rigidity equals the torsional rigidity of the unreinforced shaft given by $\frac{\pi}{2} \mathrm{G}_{m} R^{4}$ see Chen, Benveniste and Chuang (2002). A recent summary of results involving neutral inclusions in the context of the theory of effective properties is given in Milton (2002).

In this article we examine the effect of the coating phase on the torsional rigidity of coated fiber reinforced shafts. We build on the previous results and develop a variational methodology to assess the optimality or suboptimality of coated fiber configurations. Here the cross section of each coated fiber is taken to be circular and the radius of the $i^{\text {th }}$ fiber cross section is denoted by $a_{i}$ and the outer radius of the coating is given by $b_{i}$. The union of the coated fibers is denoted by $A$. The remaining part of the cross section containing matrix material is denoted by $A_{m}$. The shaft cross section is denoted by $\Omega$ and $\Omega=A \cup A_{m}$. The results given in this paper follow easily from a set of bounds on the torsional rigidity derived using the variational principles given by (2.1) and (2.2).

We provide a brief outline of the bounds derived in this paper. Upper and lower bounds on the torsional rigidity for shafts with circular cross section reinforced with coated fibers are given in Proposition 2.1. These bounds are given in terms of the effective shear moduli and torsional rigidity of each coated fiber. Next we consider shafts with arbitrary simply connected cross section. Here upper bounds are given in terms of the polar moment of inertia of the shaft cross section $I_{0}(\Omega)$ and the effective shear moduli and torsional rigidity of each coated fiber, see Proposition 3.1. If in addition one knows that $\mathrm{G}_{c}^{i} \leq \mathrm{G}_{f}^{i}$, for $i=1, \ldots, N$, then it is shown that one can derive a tighter upper bound given in terms of the torsional rigidity $\mathcal{T}_{0}(\Omega)$ of the shaft cross section and the effective shear moduli and torsional rigidity of each coated fiber, see Proposition 5.2. When $\mathrm{G}_{c}^{i} \geq \mathrm{G}_{f}^{i}$, for $i=1, \ldots, N$, a lower bound is derived and is given in terms of $\mathcal{T}_{0}(\Omega)$ and the effective shear moduli and torsional rigidity of each coated fiber, see Proposition 6.2.

The bounds are used to establish the three reinforcement inequalities and three geometric inequalities presented in Section 2. The reinforcement inequalities provide explicit criteria that determine when the torsional rigidity of a single coated fiber centered inside a shaft with circular cross section is either optimal or suboptimal among all coated fiber configurations for shafts with cross sections satisfying prescribed isoperimetric constraints, see Propositions 2.2, 2.3 and 2.4. The geometric inequalities provide explicit criteria that determine when the torsional rigidity of the coated fiber reinforced shaft is either greater than or less than the torsional rigidity of the same shaft in the absence of the coated fiber reinforcement, see Propositions 2.5, 2.6 and 2.7. In all cases the optimality conditions are expressed in terms of the effective shear modulus and torsional rigidity of each coated fiber.

2. Inequalities on the torsional rigidity. We begin by introducing the variational formulations for the torsional rigidity used in the subsequent analysis. The torsional rigidity for a system of $N$ coated fibers inside a shaft with cross section $\Omega$ is denoted by $\mathcal{T}^{N}(\Omega)$. Points inside $\Omega$ are denoted by $\mathbf{x}=\left(x_{1}, x_{2}\right)$ and the coordinate system is chosen such that the origin lies inside $\Omega$. The first variational principle is given in terms of virtual stress potentials $\varphi$ that vanish on the boundary of the shaft cross section that are square integrable and have square integrable gradients. It is 
given by

$$
\mathcal{T}^{N}(\Omega)=-2 \min _{\varphi}\left\{\frac{1}{2} \int_{\Omega} G^{-1}(\mathbf{x})|\nabla \varphi|^{2} d \mathbf{x}-2 \int_{\Omega} \varphi d \mathbf{x}\right\}
$$

where the piecewise constant shear modulus $G(\mathbf{x})$ is $\mathrm{G}_{m}$ in the matrix and takes the values $\mathrm{G}_{f}^{i}$ and $\mathrm{G}_{c}^{i}$ in the $i^{\text {th }}$ fiber and coating respectively. Next we define the vector $\mathbf{x}^{\perp}$ to be given by $\left(-x_{2}, x_{1}\right)$. The second variational principle is given in terms of virtual warping functions $\tilde{w}$ that are square integrable and have square integrable gradients. It is given by

$$
\mathcal{T}^{N}(\Omega)=\min _{\tilde{w}}\left\{\int_{\Omega} G(\mathbf{x})\left|\nabla \tilde{w}+\mathbf{x}^{\perp}\right|^{2} d \mathbf{x}\right\} .
$$

Motivated by (1.4) and (1.5) we start by considering shafts with circular cross section of radius $R$. For this case we denote the shaft cross section by $D_{R}$. The torsional rigidity of $D_{R}$ reinforced with $N$ coated fibers is written as $\mathcal{T}^{N}\left(D_{R}\right)$. Here the coordinates are chosen such that the center of the shaft is the origin. The method presented here is simple. The trial fields are designed so that they become the actual stress potential or warping field in the composite when $G_{C C A}^{i}=\mathrm{G}_{m}$, for $i=1, \ldots, N$. Otherwise these fields are admissible trials and when substituted into the variational principles give upper and lower bounds on the torsional rigidity. In this way the upper and lower bounds match when $G_{C C A}^{i}=\mathrm{G}_{m}$, for $i=1, \ldots, N$. For a system of $N$ coated fibers with centers located at the points $\mathbf{x}_{i}, i=1, \ldots, N$ the bounds are given by the following Proposition.

PROPOSITION 2.1.

$\mathcal{A}^{N}+\pi \sum_{i=1}^{N}\left|\mathbf{x}_{i}\right|^{2} b_{i}^{2} \frac{\mathrm{G}_{m}}{G_{C C A}^{i}}\left(G_{C C A}^{i}-\mathrm{G}_{m}\right) \leq \mathcal{T}^{N}\left(D_{R}\right) \leq \mathcal{A}^{N}+\pi \sum_{i=1}^{N}\left|\mathbf{x}_{i}\right|^{2} b_{i}^{2}\left(G_{C C A}^{i}-\mathrm{G}_{m}\right)$,

where $\mathcal{A}^{N}$ is given by (1.5). The upper and lower bound agree when $G_{C C A}^{i}=\mathrm{G}_{m}$, for $i=1, \ldots, N$. These upper and lower bounds are derived in Sections 3 and 4 respectively.

In what follows we apply Proposition 2.1 to obtain the basic reinforcement inequality for shafts of circular cross section reinforced with a finite number $N$ of coated fibers. Here we suppose that the shear moduli of each fiber and coating are the same, i.e., $\mathrm{G}_{f}^{i}=\mathrm{G}_{f}$ and $\mathrm{G}_{c}^{i}=\mathrm{G}_{c}$. In addition it is supposed that the ratio of outer and inner coating radius is the same for each coated fiber, i.e., $\nu_{i}=\nu, i=1, \ldots, N$. For this case $G_{C C A}^{i}=G_{C C A}$ where

$$
G_{C C A}=\mathrm{G}_{c}\left(\frac{\mathrm{G}_{c}(1-\nu)+\mathrm{G}_{f}(1+\nu)}{\mathrm{G}_{c}(1+\nu)+\mathrm{G}_{f}(1-\nu)}\right)
$$

and for $G_{C C A}=\mathrm{G}_{m}$ the torsional rigidity given by (1.5) becomes

$$
\mathcal{A}^{N}=\overline{\mathcal{A}}=\frac{\pi}{2}\left(\mathrm{G}_{m} R^{4}-\mathrm{G}_{m} \bar{b}^{4}+\mathrm{G}_{c} \bar{b}^{4}\left(1-\nu^{2}\right)+\mathrm{G}_{f} \nu^{2} \bar{b}^{4}\right),
$$

where $\bar{b}^{4}=\sum_{i=1}^{N} b_{i}^{4}$. Here $\overline{\mathcal{A}}$ is precisely the torsional rigidity of a single coated fiber with outer coating radius $\bar{b}$ and fiber radius $\bar{a}=\nu^{1 / 2} \bar{b}$ when the centers of the coated fiber cross section and shaft cross section are the same. The torsional rigidity of the 
concentric coated fiber shaft configuration is given by the right hand side of (2.5) for all values of $\mathrm{G}_{m}, \mathrm{G}_{f}, \mathrm{G}_{c}$ and $\bar{a} \leq \bar{b} \leq R$. We note here that the area of the fiber cross section is given by $\pi \bar{a}^{2}=\pi \sqrt{\sum_{i=1}^{N} a_{i}^{4}}$.

The following reinforcement inequalities follow from Proposition 2.1 and gives conditions for which the concentric coated fiber and circular shaft cross section is either optimal or suboptimal.

Proposition 2.2. Reinforcement Inequalities I.

If $G_{C C A} \leq \mathrm{G}_{m}$, then the torsional rigidity associated with $N$ coated fibers is less than or equal to the rigidity associated with a single centered circular coated fiber with fiber radius $\bar{a}=\nu^{1 / 2} \bar{b}$, i.e.,

$$
\mathcal{T}^{N}\left(D_{R}\right) \leq \overline{\mathcal{A}} .
$$

Otherwise if $G_{C C A} \geq \mathrm{G}_{m}$, then the torsional rigidity associated with $N$ coated fibers is greater than or equal to that of a single centered circular coated fiber with fiber radius $\bar{a}=\nu^{1 / 2} \bar{b}$, i.e.,

$$
\mathcal{T}^{N}\left(D_{R}\right) \geq \overline{\mathcal{A}} .
$$

These inequalities are independent of the number and location of the coated fibers.

When all fibers have the same radius $a$ and coating thickness $\ell$ one easily passes to the distinguished limit given by (1.6) in Proposition 2.2 to recover Theorem 1.3 of Lipton (1998) for imperfectly bonded fiber reinforced shafts.

Next we consider the more general case where the shaft can have arbitrary simply connected cross section $\Omega$. Here we consider all configurations of $N$ coated fibers with prescribed fiber radii $a_{i}, i=1, \ldots, N$ and consider all cross sections $\Omega$ with prescribed polar moment of inertia. We apply the upper bound on the torsional rigidity given by Proposition 3.1 to obtain

PROPOSITION 2.3. Reinforcement Inequality II.

Consider any shaft with polar moment of inertia with respect to the origin equal to $\pi R^{4} / 2$ reinforced with $N$ circular coated fibers. If $G_{C C A} \leq \mathrm{G}_{m}$, then the torsional rigidity $\mathcal{T}^{N}(\Omega)$ is less than or equal to the torsional rigidity associated with a shaft with circular cross section of radius $R$ reinforced with a single centered circular coated fiber with fiber radius $\bar{a}$ given by

$$
\pi \bar{a}^{2}=\pi \sqrt{\sum_{i=1}^{N} a_{i}^{4}}
$$

and $\bar{b}=\nu^{-1 / 2} \bar{a}$.

When $\mathrm{G}_{c} \leq \mathrm{G}_{f}$ we can appeal to the tighter upper bound on the torsional rigidity given by Proposition 5.2 to obtain a reinforcement inequality that holds for all shaft cross sections $\Omega$ with prescribed cross-sectional area.

Proposition 2.4. Reinforcement Inequality III.

Consider any shaft with cross-sectional area equal to $\pi R^{2}$ reinforced with $N$ circular coated fibers. If $G_{C C A} \leq \mathrm{G}_{m}$, and $\mathrm{G}_{c} \leq \mathrm{G}_{f}$ then the torsional rigidity $\mathcal{T}^{N}(\Omega)$ is less than or equal to the torsional rigidity associated with a shaft with circular cross section of radius $R$ reinforced with a single centered circular coated fiber with fiber radius $\bar{a}$ given by

$$
\pi \bar{a}^{2}=\pi \sqrt{\sum_{i=1}^{N} a_{i}^{4}}
$$


and $\bar{b}=\nu^{-1 / 2} \bar{a}$.

It is evident from the inequality $\sqrt{\sum_{i=1}^{N} a_{i}^{4}} \leq \sum_{i=1}^{N} a_{i}^{2}$ that the cross-sectional area of the single centered circular fiber appearing in Propositions 2.2, 2.3 and 2.4 is less than or equal to the joint cross-sectional area of the $N$ fibers.

Now we consider the more general case where the shear moduli of the fiber and coating and the ratio of the inner radius and outer radius of the coating are allowed to differ between coated fibers. In this context we present explicit conditions on the effective shear modulus and torsional rigidity of each coated fiber that shows when the torsional rigidity of the coated fiber reinforced shaft is either greater or less than the torsional rigidity of the shaft without reinforcement.

For shafts with circular cross sections of radius $R$, i.e., $\Omega=D_{R}$, we have the following

Proposition 2.5. Geometric Inequalities I. If $\sum_{i=1}^{N} T_{f}^{i} \leq \sum_{i=1}^{N} \frac{\pi}{2} \mathrm{G}_{m} b_{i}^{4}$ and $G_{C C A}^{i} \leq \mathrm{G}_{m}$, then

$$
\mathcal{T}^{N}\left(D_{R}\right) \leq \frac{\pi}{2} \mathrm{G}_{m} R^{4}
$$

If $\sum_{i=1}^{N} T_{f}^{i} \geq \sum_{i=1}^{N} \frac{\pi}{2} \mathrm{G}_{m} b_{i}^{4}$ and $G_{C C A}^{i} \geq \mathrm{G}_{m}$, then

$$
\mathcal{T}^{N}\left(D_{R}\right) \geq \frac{\pi}{2} \mathrm{G}_{m} R^{4} .
$$

The inequalities (2.10) and (2.11) are independent of the number and location of the coated fibers. These inequalities follow immediately from Proposition 2.1.

Now we extend these results to simply connected cross sections $\Omega$ and denote the torsional rigidity for simply connected shaft cross sections with shear modulus unity by $\mathcal{T}_{0}(\Omega)$. The following geometric inequality shows when a system of coated fibers always decreases the torsional rigidity below that of the unreinforced shaft.

Proposition 2.6. Geometric Inequality II.

Suppose that $\mathrm{G}_{c}^{i} \leq \mathrm{G}_{f}^{i}, i=1, \ldots, N$. If $\sum_{i=1}^{N} T_{f}^{i} \leq \sum_{i=1}^{N} \frac{\pi}{2} \mathrm{G}_{m} b_{i}^{4}$ and $G_{C C A}^{i} \leq \mathrm{G}_{m}$, then

$$
\mathcal{T}^{N}(\Omega) \leq \mathrm{G}_{m} \mathcal{T}_{0}(\Omega)
$$

Equality holds in (2.12) when the shaft cross section is circular, $\sum_{i=1}^{N} T_{f}^{i}=\sum_{i=1}^{N} \frac{\pi}{2} \mathrm{G}_{m} b_{i}^{4}$ and $G_{C C A}^{i}=\mathrm{G}_{m}$ for $i=1, \ldots, N$. This result follows from the upper bound on the torsional rigidity given by Proposition 5.2.

The following geometric inequality shows when a system of coated fibers always increases the torsional rigidity above that of the unreinforced shaft.

Proposition 2.7. Geometric Inequality III.

Suppose that $\mathrm{G}_{c}^{i} \geq \mathrm{G}_{f}^{i}, i=1, \ldots, N$. If $\sum_{i=1}^{N} T_{f}^{i} \geq \sum_{i=1}^{N} \frac{\pi}{2} \mathrm{G}_{m} b_{i}^{4}$ and $G_{C C A}^{i} \geq \mathrm{G}_{m}$, then

$$
\mathcal{T}^{N}(\Omega) \geq \mathrm{G}_{m} \mathcal{T}_{0}(\Omega)
$$

Equality holds in (2.13) when the shaft cross section is circular, $\sum_{i=1}^{N} T_{f}^{i}=\sum_{i=1}^{N} \frac{\pi}{2} \mathrm{G}_{m} b_{i}^{4}$ and $G_{C C A}^{i}=\mathrm{G}_{m}$ for $i=1, \ldots, N$. This result follows from the lower bound on the torsional rigidity given by Proposition 6.2. 
3. Upper bounds on the torsional rigidity for shafts reinforced with circular coated fibers. In this Section we develop trial warping functions for configurations of circular coated fibers. These are substituted into the variational principle (2.2) and deliver the upper bound presented in Proposition 2.1. The trial warping functions constructed here will be admissible for shaft cross sections of any shape. For circular shaft cross sections it is shown that the trial warping functions become the actual warping displacement in the shaft when $G_{C C A}^{i}=\mathrm{G}_{m}$ for $i=1, \ldots, N$.

Consider a shaft of arbitrary cross section $\Omega$ reinforced with $N$ circular coated fibers with centers at the points $\mathbf{x}_{i}, i=1, \ldots, N$. The radius of the $i^{\text {th }}$ fiber is $a_{i}$ and the outer radius of the coated fiber is $b_{i}$. The coating occupies the annular shell with inner and outer radii $a_{i}$ and $b_{i}$. The trial warping function $\tilde{w}$ is chosen such that $\tilde{w}=0$ outside the coated fibers. In each coated fiber the function $\tilde{w}$ is required to be harmonic inside the fiber and harmonic inside the coating. It is required that $\tilde{w}$ be continuous across the interface separating the fiber and coating and

$$
\mathrm{G}_{f}^{i}\left(\nabla \tilde{w}_{\left.\right|_{f}}+\mathbf{x}^{\perp}\right) \cdot \mathbf{n}=\mathrm{G}_{c}^{i}\left(\nabla \tilde{w}_{\left.\right|_{c}}+\mathbf{x}^{\perp}\right) \cdot \mathbf{n}
$$

across the fiber-coating interface. Here the subscripts indicate the side of the interface over which the quantities are evaluated and $\mathbf{n}$ is the outward directed unit normal in the fiber coating interface. The final requirement is that $\tilde{w}$ vanish on the boundary of the coated fiber. It is clear that the continuity conditions for $\tilde{w}$ at material interfaces ensure that it is an admissible trial field for (2.2).

We solve the transmission boundary value problem inside each coated fiber to obtain the explicit formula for $\tilde{w}$. The polar coordinates $(\theta, r)$ are chosen such that the axis $\theta=0$ coincides with the direction given by $\mathbf{x}^{\perp}{ }_{i}$ and origin with $\mathbf{x}_{i}$. In these coordinates, the transmission condition (3.1) on the $i^{\text {th }}$ fiber-coating interface becomes

$$
\mathrm{G}_{c}^{i} \partial_{r} \tilde{w}_{\left.\right|_{c}}-\mathrm{G}_{f}^{i} \partial_{r} \tilde{w}_{\left.\right|_{f}}=\left(\mathrm{G}_{f}^{i}-\mathrm{G}_{c}^{i}\right)\left|\mathbf{x}_{i}\right| \cos \theta \text {, on } r=a_{i} .
$$

Since $\tilde{w}$ is required to be harmonic inside each fiber and coating it follows that

$$
\tilde{w}=C_{1} r \cos \theta, \text { for } r \leq a_{i},
$$

and

$$
\tilde{w}=\left(C_{2} r+C_{3} r^{-1}\right) \cos \theta, \text { for } a_{i} \leq r \leq b_{i} .
$$

The transmission conditions at $r=a_{i}$ and boundary condition at $r=b_{i}$ require that

$$
\begin{aligned}
C_{2} b_{i}^{2}+C_{3} & =0 \\
C_{1} a_{i}^{2}-C_{2} a_{i}^{2}-C_{3} & =0 \\
\mathrm{G}_{c}^{i} a_{i}^{2} C_{2}-\mathrm{G}_{c}^{i} C_{3}-\mathrm{G}_{f}^{i} a_{i}^{2} C_{1} & =\left(\mathrm{G}_{f}^{i}-\mathrm{G}_{c}^{i}\right) a_{i}^{2}\left|\mathbf{x}_{i}\right| .
\end{aligned}
$$

Solution of (3.5) shows that inside each coated fiber the trial warping function is given by

$$
\tilde{w}=C_{1}^{i} r \cos \theta, \text { for } r=\left|\mathbf{x}-\mathbf{x}_{i}\right| \leq a_{i}
$$

and

$$
\tilde{w}=\left(C_{2}^{i} r+C_{3}^{i} r^{-1}\right) \cos \theta, \text { for } a_{i} \leq r=\left|\mathbf{x}-\mathbf{x}_{i}\right| \leq b_{i} .
$$


Where $\Delta_{i}=\mathrm{G}_{c}^{i}\left(a_{i}^{2}+b_{i}^{2}\right)+\mathrm{G}_{f}^{i}\left(b_{i}^{2}-a_{i}^{2}\right)$ and

$$
\begin{aligned}
& C_{1}^{i}=\left(\mathrm{G}_{c}^{i}-\mathrm{G}_{f}^{i}\right)\left|\mathbf{x}_{i}\right|\left(b_{i}^{2}-a_{i}^{2}\right) / \Delta_{i} \\
& C_{2}^{i}=\left(\mathrm{G}_{f}^{i}-\mathrm{G}_{c}^{i}\right)\left|\mathbf{x}_{i}\right| a_{i}^{2} / \Delta_{i} \\
& C_{3}^{i}=\left(\mathrm{G}_{c}^{i}-\mathrm{G}_{f}^{i}\right)\left|\mathbf{x}_{i}\right| b_{i}^{2} a_{i}^{2} / \Delta_{i}
\end{aligned}
$$

Outside the coated fibers $\tilde{w}=0$.

The polar moment of inertia of the shaft cross section $\Omega$ with respect to the origin is written $I_{0}(\Omega)$. Here $I_{0}(\Omega)=\int_{\Omega}|\mathbf{x}|^{2} d \mathbf{x}$. Substitution of $\tilde{w}$ into (2.2) delivers the upper bound given in the following

Proposition 3.1. Upper bound on rigidity for arbitrary shaft cross section.

$$
\begin{aligned}
\mathcal{T}^{N}(\Omega) & \leq \mathrm{G}_{m} I_{0}(\Omega)+\sum_{i=1}^{N}\left(\frac{\pi}{2} T_{f}^{i}-\frac{\pi}{2} \mathrm{G}_{m} b_{i}^{4}\right) \\
& +\pi \sum_{i=1}^{N}\left|\mathbf{x}_{i}\right|^{2} b_{i}^{2}\left(G_{C C A}^{i}-\mathrm{G}_{m}\right) .
\end{aligned}
$$

Next we consider shafts with circular cross section of radius $R$. In order for the trial warping field $\tilde{w}$ to be the actual warping displacement in the shaft it must also satisfy the transmission condition on the coating-matrix interface $\left|\mathbf{x}-\mathbf{x}_{i}\right|=b_{i}$ given by

$$
\mathrm{G}_{m} \mathbf{x}^{\perp} \cdot \mathbf{n}=\mathrm{G}_{c}^{i}\left(\nabla \tilde{w}_{\left.\right|_{c}}+\mathbf{x}^{\perp}\right) \cdot \mathbf{n} .
$$

This gives the extra condition

$$
\mathrm{G}_{c}^{i} C_{2}^{i} b_{i}^{2}-\mathrm{G}_{c}^{i} C_{3}^{i}=\left(\mathrm{G}_{m}-\mathrm{G}_{c}^{i}\right)\left|\mathbf{x}_{i}\right| b_{i}^{2} .
$$

This condition together with the conditions given by (3.5) provide an over determined system of equations for the coefficients $C_{1}^{i}, C_{2}^{i}, C_{3}^{i}$ in each coated fiber. It is easily seen that the over determined system has a solution when $G_{C C A}^{i}=\mathrm{G}_{m}$. For this case the function $\tilde{w}$ becomes the warping displacement in the shaft and we recover the formula

$$
\mathcal{T}^{N}\left(D_{R}\right)=\mathcal{A}^{N}
$$

where $\mathcal{A}^{N}$ is given by (1.5).

4. Lower bounds on the torsional rigidity for circular shafts reinforced with circular coated fibers. In this Section we develop trial stress potentials for configurations of circular coated fibers. These are substituted into the variational principle (2.1) to obtain the lower bound given in Proposition 2.1.

We consider a circular shaft cross section of radius $R$ reinforced with $N$ coated fibers. Outside the coated fibers the trial stress potential $\varphi$ is taken to be $\varphi=$ $\frac{1}{2} \mathrm{G}_{m}\left(R^{2}-|\mathbf{x}|^{2}\right)$. The trial potential is taken to be continuous across the matrixcoating interface specified by $\left|\mathbf{x}-\mathbf{x}_{i}\right|=b_{i}$. It is easily seen that

$$
\varphi=h(\mathbf{x})=-\mathrm{G}_{m}\left(\mathbf{x}-\mathbf{x}_{i}\right) \cdot \mathbf{x}_{i}+\frac{1}{2} \mathrm{G}_{m}\left(R^{2}-b_{i}^{2}-\left|\mathbf{x}_{i}\right|^{2}\right)
$$

on this interface in view of the condition $\left|\mathbf{x}-\mathbf{x}_{i}\right|=b_{i}$. The trial is taken to be continuous inside the coated fiber and is given by $\varphi=\psi^{i}+r^{i}$ in the $i^{\text {th }}$ fiber. Here 
$\psi^{i}$ is chosen to be the stress potential generated inside the coated fiber when it is subject to torsion loading. It is the solution of the transmission problem inside the coated fiber given by

$$
\begin{gathered}
\mathrm{G}_{f}^{i-1}(x) \Delta \psi^{i}=-2 \text { in the fiber },\left|\mathbf{x}-\mathbf{x}_{i}\right|<a_{i}, \\
\mathrm{G}_{c}^{i-1}(x) \Delta \psi^{i}=-2 \text { in the coating, } a_{i}<\left|\mathbf{x}-\mathbf{x}_{i}\right|<b_{i},
\end{gathered}
$$

$\psi^{i}$ is continuous across the fiber-coating interface,

$$
\mathrm{G}_{f}^{i-1} \nabla \psi_{\left.\right|_{f}}^{i} \cdot \mathbf{n}=\mathrm{G}_{c}^{i-1} \nabla \psi_{\left.\right|_{c}}^{i} \cdot \mathbf{n} \text { on }\left|\mathbf{x}-\mathbf{x}_{i}\right|=a_{i}
$$

and $\psi^{i}=0$ on $\left|\mathbf{x}-\mathbf{x}_{i}\right|=b_{i}$. It is easily seen that $\psi^{i}$ is given by

$$
\begin{aligned}
\psi^{i} & =-\frac{1}{2}\left(\mathrm{G}_{f}^{i}\left|\mathbf{x}-\mathbf{x}_{i}\right|^{2}-\mathrm{G}_{c}^{i}\left(b_{i}^{2}-a_{i}^{2}\right)-\mathrm{G}_{f}^{i} a_{i}^{2}\right), \text { for },\left|\mathbf{x}-\mathbf{x}_{i}\right|<a_{i}, \\
\psi^{i} & =-\frac{1}{2}\left(\mathrm{G}_{c}^{i}\left|\mathbf{x}-\mathbf{x}_{i}\right|^{2}-\mathrm{G}_{c}^{i} b_{i}^{2}\right), \text { for }, a_{i}<\left|\mathbf{x}-\mathbf{x}_{i}\right|<b_{i} .
\end{aligned}
$$

The function $r^{i}=h$ on the coating-matrix interface and is continuous inside the coated fiber. It is the solution to the transmission problem given by

$$
\begin{gathered}
\mathrm{G}_{f}^{i-1}(x) \Delta r^{i}=0 \text { in the fiber },\left|\mathbf{x}-\mathbf{x}_{i}\right|<a_{i}, \\
\mathrm{G}_{c}^{i-1}(x) \Delta r^{i}=0 \text { in the coating }, a_{i}<\left|\mathbf{x}-\mathbf{x}_{i}\right|<b_{i}
\end{gathered}
$$

and

$$
\mathrm{G}_{f}^{i-1} \nabla r_{\left.\right|_{f}}^{i} \cdot \mathbf{n}=\mathrm{G}_{c}^{i-1} \nabla r_{\left.\right|_{c}}^{i} \cdot \mathbf{n} \text { on }\left|\mathbf{x}-\mathbf{x}_{i}\right|=a_{i} .
$$

In the polar coordinates $(\theta, r)$ chosen such that the axis $\theta=0$ coincides with the vector $\mathbf{x}_{i}$ and $r=\left|\mathbf{x}_{i}-\mathbf{x}\right|$, the solution of the transmission problem for $r^{i}$ is given by

$$
\begin{aligned}
& r^{i}=C_{1}^{i} r \cos \theta+k^{i}, \text { for },\left|\mathbf{x}-\mathbf{x}_{i}\right|<a_{i}, \\
& r^{i}=\left(C_{2}^{i} r+C_{3}^{i} r^{-1}\right) \cos \theta+k^{i}, \text { for }, a_{i}<\left|\mathbf{x}-\mathbf{x}_{i}\right|<b_{i},
\end{aligned}
$$

where

$$
\begin{aligned}
k^{i} & =\frac{\mathrm{G}_{m}}{2}\left(R^{2}-b_{i}^{2}-\left|\mathbf{x}_{i}\right|^{2}\right) \\
C_{1}^{i} & =-\mathrm{G}_{m}\left|\mathbf{x}_{i}\right| b_{i}^{2} 2 \mathrm{G}_{f}^{i} / D_{i} \\
C_{2}^{i} & =-\mathrm{G}_{m}\left|\mathbf{x}_{i}\right| b_{i}^{2}\left(\mathrm{G}_{f}^{i}+\mathrm{G}_{c}^{i}\right) / D_{i} \\
C_{3}^{i} & =-\mathrm{G}_{m}\left|\mathbf{x}_{i}\right| a_{i}^{2} b_{i}^{2}\left(\mathrm{G}_{f}^{i}-\mathrm{G}_{c}^{i}\right) / D_{i},
\end{aligned}
$$

and $D_{i}=\left(b_{i}^{2}+a_{i}^{2}\right) \mathrm{G}_{f}^{i}+\left(b_{i}^{2}-a_{i}^{2}\right) \mathrm{G}_{c}^{i}$. The lower bound in (2.3) follows from substitution of this trial potential into the variational principle (2.1).

In order for the trial potential field $\varphi$ to be the actual stress potential in the shaft it must also satisfy the transmission condition on the coating-matrix interface given by

$$
\mathrm{G}_{m}^{-1} \nabla \varphi_{\left.\right|_{m}} \cdot \mathbf{n}=\mathrm{G}_{c}^{i-1}\left(\nabla \psi_{\left.\right|_{c}}^{i}+\nabla r_{\left.\right|_{c}}^{i}\right) \cdot \mathbf{n}
$$


Substitution and working in polar coordinates shows that (4.11) gives the extra condition

$$
\mathrm{G}_{c}^{i-1}\left(C_{2}^{i}-C_{3}^{i} b_{i}^{-2}\right)=-\left|\mathbf{x}_{i}\right| .
$$

This condition together with the system of equations (4.10) over determines the coefficients $C_{1}^{i}, C_{2}^{i}, C_{3}^{i}$ in each coated sphere. It is easily seen that the over determined system has a solution when $G_{C C A}^{i}=\mathrm{G}_{m}$. For this case the function $\varphi$ becomes the stress potential in the shaft and we recover the formula

$$
\mathcal{T}^{N}\left(D_{R}\right)=\mathcal{A}^{N}
$$

where $\mathcal{A}^{N}$ is given by (1.5).

5. Upper bounds on the torsional rigidity for $\mathrm{G}_{c}^{i} \leq \mathrm{G}_{f}^{i}$. In this section we focus on the case where $\mathrm{G}_{c}^{i} \leq \mathrm{G}_{f}^{i}, i=1, \ldots, N$. Here we are able to get tighter upper bounds on the torsional rigidity for shaft cross sections of arbitrary shape. Our approach follows the methodology developed in Lipton (1998). We fix the crosssection of the shaft $\Omega$ and investigate the effects of adding a circular coated fiber to an already existing configuration of $N-1$ coated fibers. At present no assumptions on the geometry or shear moduli of the $N-1$ coated fibers are made. We denote the part of the shaft cross-section already occupied by the coated fibers by $A$ and the cross-section of the circular coated fiber to be added by $\Sigma$. Here $\Sigma$ is composed of a circular fiber of radius $a_{N}$ with shear modulus $\mathrm{G}_{f}^{N}$ surrounded by a coating of outer radius $b_{N}$ with shear modulus $\mathrm{G}_{c}^{N}$. The torsional rigidity of the original configuration is denoted by $\mathcal{T}(A, \Omega)$. The rigidity associated with the added fiber is written as $\mathcal{T}(A \cup \Sigma, \Omega)$. We recall that the torsional rigidity obtained by replacing coating and fiber shear moduli with the matrix shear moduli in $\Sigma$ is given by $\frac{\pi}{2} \mathrm{G}_{m} b_{N}^{4}$. Here $b_{N}$ is the outer radius of the coating. The torsional rigidity of the coated fiber is $T_{f}^{N}=\frac{\pi}{2}\left(\mathrm{G}_{c}^{N}\left(b_{N}^{4}-a_{N}^{4}\right)+\mathrm{G}_{f}^{N} a_{N}^{4}\right)$. We state the following:

PROPOSITION 5.1. Upper rigidity inequality

If $\mathrm{G}_{c}^{N} \leq \mathrm{G}_{f}^{N}$ and if

$$
G_{C C A}^{N} \leq \mathrm{G}_{m}
$$

then

$$
\mathcal{T}(A \cup \Sigma, \Omega) \leq \mathcal{T}(A, \Omega)+T_{f}^{N}-\frac{\pi}{2} \mathrm{G}_{m} b_{N}^{4}
$$

Proposition 5.1 is established with the aid of the variational principle given by (2.1). We remark that the methods used to establish this inequality apply to the case when the fiber cross-section is multiply connected. One writes $(2.1)$ as $\mathcal{T}(A, \Omega)=$ $-2 \mathcal{E}(A, \Omega)$ where

$$
\mathcal{E}(A, \Omega)=\min _{\varphi}\left\{\frac{1}{2} \int_{\Omega} G^{-1}(\mathbf{x})|\nabla \varphi|^{2} d \mathbf{x}-2 \int_{\Omega} \varphi d \mathbf{x}\right\} .
$$

Here the piecewise constant shear modulus $G(\mathbf{x})$ takes the value $\mathrm{G}_{m}$ in the matrix and takes the values $\mathrm{G}_{f}^{i}$ and $\mathrm{G}_{c}^{i}$ in the $i^{\text {th }}$ fiber and coating respectively. The idea of the proof is to estimate the quantity $\mathcal{E}(A, \Omega)$ in terms of $\mathcal{E}(A \cup \Sigma, \Omega)$ associated 
with the additional fiber. We let $\mathcal{G}(\mathbf{x})$ denote the piece wise constant shear modulus for the configuration $A \cup \Sigma$. Here $\mathcal{G}(\mathbf{x})=G(\mathbf{x})$ outside of $\Sigma$ and inside $\Sigma$ the shear modulus $\mathcal{G}(\mathbf{x})=\mathrm{G}_{f}^{N}$ in the fiber and $\mathcal{G}(\mathbf{x})=\mathrm{G}_{c}^{N}$ in the coating. We regroup terms in the variational principle (5.3) and write:

$$
\begin{aligned}
\mathcal{E}(A, \Omega) & =\min _{\varphi}\left\{\frac{1}{2} \int_{\Omega} \mathcal{G}^{-1}(\mathbf{x})|\nabla \varphi|^{2} d \mathbf{x}-2 \int_{\Omega} \varphi d \mathbf{x}+\right. \\
& +\frac{1}{2}\left(\int_{\Sigma}\left(\mathrm{G}_{m}^{-1}-\mathcal{G}^{-1}(\mathbf{x})\right)|\nabla \varphi|^{2} d \mathbf{x}\right\} .
\end{aligned}
$$

We obtain an estimate by substitution of a suitable trial field in (5.4). Our choice is made as follows. We introduce the stress potential $\tilde{\Phi}$ for the configuration $A \cup \Sigma$. Here $\tilde{\Phi}$ is continuous in $\Omega$,

$$
-\mathcal{G}^{-1}(\mathbf{x}) \Delta \tilde{\Phi}=2
$$

and satisfies the transmission conditions

$$
\mathrm{G}_{f}^{i-1} \nabla \tilde{\Phi}_{\left.\right|_{f}} \cdot \mathbf{n}=\mathrm{G}_{c}^{i-1} \nabla \tilde{\Phi}_{\left.\right|_{c}} \cdot \mathbf{n} \text { on the fiber-coating interface, }\left|\mathbf{x}-\mathbf{x}_{i}\right|=a_{i}
$$

and

$$
\mathrm{G}_{f}^{i-1} \nabla \tilde{\Phi}_{\left.\right|_{c}} \cdot \mathbf{n}=\mathrm{G}_{m}{ }^{-1} \nabla \tilde{\Phi}_{\left.\right|_{m}} \cdot \mathbf{n} \text { on the matrix-coating interface, }\left|\mathbf{x}-\mathbf{x}_{i}\right|=b_{i} .
$$

The trial field $\varphi$ is chosen to match $\tilde{\Phi}$ outside the coated fiber cross-section $\Sigma$ but inside we suppose that $\varphi=\tilde{\Phi}+\delta$. Where $\delta$ is continuous, vanishes on the boundary of $\Sigma$, is square integrable inside $\Sigma$ and has square integrable gradient over $\Sigma$. One easily checks that

$$
\int_{\Sigma} \mathcal{G}^{-1}(\mathbf{x}) \nabla \tilde{\Phi} \cdot \nabla \delta d \mathbf{x}=2 \int_{\Sigma} \delta d \mathbf{x}
$$

Substitution of $\varphi$ into (5.4) gives:

$$
\begin{aligned}
\mathcal{E}(A, \Omega) & \leq \frac{1}{2}\left(\int_{\Omega / \Sigma} \mathcal{G}^{-1}(\mathbf{x})|\nabla \tilde{\Phi}|^{2} d \mathbf{x}+\int_{\Sigma} \mathcal{G}^{-1}(\mathbf{x})|\nabla \tilde{\Phi}+\nabla \delta|^{2} d \mathbf{x}\right) \\
& -2 \int_{\Omega} \tilde{\Phi} d \mathbf{x}-2 \int_{\Sigma} \delta d \mathbf{x} \\
& +\frac{1}{2}\left(\int_{\Sigma}\left(\mathrm{G}_{m}^{-1}-\mathcal{G}^{-1}(\mathbf{x})\right)|\nabla \varphi|^{2} d \mathbf{x} .\right.
\end{aligned}
$$

We apply (5.8) and expand the second term on the right-hand side of (5.9) to find:

$$
\int_{\Sigma} \mathcal{G}^{-1}(\mathbf{x})|\nabla \tilde{\Phi}+\nabla \delta|^{2} d \mathbf{x}=\int_{\Sigma} \mathcal{G}^{-1}(\mathbf{x})|\nabla \tilde{\Phi}|^{2} d \mathbf{x}+\int_{\Sigma} \mathcal{G}^{-1}(\mathbf{x})|\nabla \delta|^{2} d \mathbf{x}+4 \int_{\Sigma} \delta d \mathbf{x}
$$

Substitution of (5.10) into (5.9) yields:

$$
\begin{aligned}
\mathcal{E}(A, \Omega) & \leq \mathcal{E}(A \cup \Sigma, \Omega)+\frac{1}{2} \int_{\Sigma} \mathcal{G}^{-1}(\mathbf{x})|\nabla \delta|^{2} d \mathbf{x}+ \\
& +\frac{1}{2}\left(\int_{\Sigma}\left(\mathrm{G}_{m}^{-1}-\mathcal{G}^{-1}(\mathbf{x})\right)|\nabla \varphi|^{2} d \mathbf{x} .\right.
\end{aligned}
$$


Multiplying by -2 and arranging terms we find that

$$
\begin{aligned}
\mathcal{T}(A \cup \Sigma, \Omega) & \leq \mathcal{T}(A, \Omega)+\int_{\Sigma} \mathcal{G}^{-1}(\mathbf{x})|\nabla \delta|^{2} d \mathbf{x}+ \\
& +\int_{\Sigma}\left(\mathrm{G}_{m}^{-1}-\mathcal{G}^{-1}(\mathbf{x})\right)|\nabla \varphi|^{2} d \mathbf{x} .
\end{aligned}
$$

Next we minimize the right-hand side of (5.12) with respect to $\delta$ to obtain

$$
\mathcal{T}(A \cup \Sigma, \Omega) \leq \mathcal{T}(A, \Omega)+\mathcal{U}
$$

where

$$
\mathcal{U}=\int_{\Sigma}\left(\mathrm{G}_{m}^{-1}-\mathcal{G}^{-1}(\mathbf{x})\right) \nabla \hat{\varphi} \cdot \nabla \tilde{\Phi} d \mathbf{x}
$$

Here $\hat{\varphi}=\hat{\delta}+\tilde{\Phi}$ in $\Sigma$ and $\hat{\delta}$ solves

$$
\int_{\Sigma} \mathrm{G}_{m}^{-1} \nabla \hat{\delta} \cdot \nabla u d \mathbf{x}=\int_{\Sigma}\left(\mathcal{G}^{-1}(\mathbf{x})-\mathrm{G}_{m}^{-1}\right) \nabla \tilde{\Phi} \cdot \nabla u d \mathbf{x},
$$

for every trial $u$ vanishing on the boundary of $\Sigma$. From (5.15) and $(5.5-5.7)$ we see that $\hat{\varphi}$ solves

$$
\int_{\Sigma} \mathrm{G}_{m}^{-1} \nabla \hat{\varphi} \cdot \nabla u d \mathbf{x}=2 \int_{\Sigma} u d \mathbf{x}
$$

for every trial $u$ vanishing on the boundary of $\Sigma$. This is equivalent to the differential equation $-\mathrm{G}_{m}^{-1} \Delta \hat{\varphi}=2$ over $\Sigma$.

We decompose the trial $\hat{\varphi}$ into two parts: $\hat{\varphi}=r+\psi^{h}$, where the function $r$ satisfies:

$$
\Delta r=0, \text { in } \Sigma \text { and } r=\tilde{\Phi} \text { on the boundary of } \Sigma,
$$

and $\psi^{h}$ satisfies:

$$
\Delta \psi^{h}=-2 \mathrm{G}_{m} \text { in } \Sigma \text { and } \psi^{h}=0 \text { on the boundary of } \Sigma \text {. }
$$

Next we decompose $\tilde{\Phi}$ into two components over $\Sigma$. We write $\tilde{\Phi}=\psi-h$. Here $\psi$ is continuous, vanishes on the boundary of $\Sigma$ and solves the torsion problem

$$
-\mathcal{G}^{-1}(\mathbf{x}) \Delta \psi=2
$$

with the transmission condition

$\left(\mathrm{G}_{f}^{N}\right)^{-1} \nabla \psi_{\left.\right|_{f}} \cdot \mathbf{n}=\left(\mathrm{G}_{c}^{N}\right)^{-1} \nabla \psi_{\left.\right|_{c}} \cdot \mathbf{n}$ on the fiber-coating interface, $\left|\mathbf{x}-\mathbf{x}_{N}\right|=a_{N}$.

The function $h$ is continuous on $\Sigma$ and $h=-\tilde{\Phi}$ on the boundary of $\Sigma$. It is the solution of

$$
-\mathcal{G}^{-1}(\mathbf{x}) \Delta h=0
$$


and $h$ satisfies the transmission condition

$\left(\mathrm{G}_{f}^{N}\right)^{-1} \nabla h_{\left.\right|_{f}} \cdot \mathbf{n}=\left(\mathrm{G}_{c}^{N}\right)^{-1} \nabla h_{\left.\right|_{c}} \cdot \mathbf{n}$ on the fiber-coating interface, $\left|\mathbf{x}-\mathbf{x}_{N}\right|=a_{N}$.

Substitution of the functions $\psi^{h}, \psi, r$, and $h$ into $\mathcal{U}$ and (5.13), gives

$$
\begin{aligned}
\mathcal{T}(A \cup \Sigma, \Omega) \leq & \mathcal{T}(A, \Omega)+T_{f}^{N}-\frac{\pi}{2} \mathrm{G}_{m} b_{N}^{4} \\
& +\int_{\Sigma} \mathrm{G}_{m}^{-1}|\nabla r|^{2} d \mathbf{x}-\int_{\Sigma} \mathcal{G}^{-1}(\mathbf{x})|\nabla h|^{2} d \mathbf{x}-4 \int_{\Sigma} r+h d \mathbf{x} .
\end{aligned}
$$

For circular fiber cross sections calculation shows that $\int_{\Sigma} r+h d \mathbf{x}=0$ and we obtain

$$
\begin{aligned}
\mathcal{T}(A \cup \Sigma, \Omega) \leq & \mathcal{T}(A, \Omega)+T_{f}^{N}-\frac{\pi}{2} \mathrm{G}_{m} b_{N}^{4} \\
& +\int_{\Sigma} \mathrm{G}_{m}^{-1}|\nabla r|^{2} d \mathbf{x}-\int_{\Sigma} \mathcal{G}^{-1}(\mathbf{x})|\nabla h|^{2} d \mathbf{x} .
\end{aligned}
$$

It is clear that Proposition 5.1 holds when the indefinite term

$$
\begin{aligned}
D & =\int_{\Sigma} \mathrm{G}_{m}^{-1}|\nabla r|^{2} d \mathbf{x}-\int_{\Sigma} \mathcal{G}^{-1}(\mathbf{x})|\nabla h|^{2} d \mathbf{x} \\
& =\mathrm{G}_{m}^{-1}\left(\int_{\Sigma}|\nabla r|^{2} d \mathbf{x}-\int_{\Sigma} \frac{\mathrm{G}_{m}}{\mathcal{G}(\mathbf{x})}|\nabla h|^{2} d \mathbf{x}\right) \leq 0 .
\end{aligned}
$$

If $\tilde{\Phi}=$ const. on the boundary of $\Sigma$ then $r=$ const. and $h=-$ const. and $D=0$. We now examine conditions for which $D \leq 0$ and $r \neq$ const. and $h \neq-$ const. To do this we search for the largest number $\beta$ for which

$$
\beta \int_{\Sigma}|\nabla r|^{2} d \mathbf{x}-\int_{\Sigma} \frac{\mathrm{G}_{m}}{\mathcal{G}(\mathbf{x})}|\nabla h|^{2} d \mathbf{x} \leq 0
$$

for every choice of $r$ and $h$ such that $h=-r$ on the boundary of $\Sigma, r$ is harmonic inside $\Sigma, h$ is harmonic in the fiber and in the coating and satisfies the transmission conditions

$$
\frac{\mathrm{G}_{m}}{\mathrm{G}_{f}^{N}} \nabla h_{\left.\right|_{f}} \cdot \mathbf{n}=\frac{\mathrm{G}_{m}}{\mathrm{G}_{c}^{N}} \nabla h_{\left.\right|_{c}} \cdot \mathbf{n},
$$

on the fiber-coating interface. The set of all such $r$ and $h$ for which $r \neq$ const. and $h \neq$ const. is denoted by $\mathcal{C}$. The largest $\beta$ is given by

$$
\hat{\beta}=\mathrm{G}_{m} \inf _{\mathcal{C}} \frac{\int_{\Sigma} \mathcal{G}(\mathbf{x})^{-1}|\nabla h|^{2} d \mathbf{x}}{\int_{\Sigma}|\nabla r|^{2} d \mathbf{x}} .
$$

The stationary values for the quotient given in (5.28) are denoted by $\beta_{n}$ and the stationary conditions for the stationary functions $\left(r_{n}, h_{n}\right)$ in $\mathcal{C}$ are given by

$$
\left(\mathrm{G}_{c}^{N}\right)^{-1} \nabla h_{n} \cdot \mathbf{n}=-\beta_{n} \nabla r_{n} \cdot \mathbf{n}
$$

on the coating matrix boundary $\left|\mathbf{x}-\mathbf{x}_{N}\right|=b_{N}$. Choosing polar coordinates $(\theta, r)$ such that the $\theta=0$ axis is along $\mathbf{x}_{N}$ and $r=\left|\mathbf{x}-\mathbf{x}_{N}\right|$ one finds that the stationary 
functions are given by

$$
\begin{aligned}
& r_{n}=K_{1}^{n} r^{n} \exp (j n \theta), \text { for } 0 \leq r \leq b_{N}, \\
& h_{n}=\left(K_{2}^{n} r^{n}\right) \exp (j n \theta) \text { for } 0 \leq r \leq a_{N}, \\
& h_{n}=\left(K_{3}^{n} r^{n}+K_{4}^{n} r^{-n}\right) \exp (j n \theta) \text { for } a_{N} \leq r \leq b_{N} .
\end{aligned}
$$

here $j=\sqrt{-1}$ and both real and imaginary parts of $r_{n}$ and $h_{n}$ are stationary functions. The constants $K_{1}^{n}$ are arbitrary and the remaining constants are given by

$$
\begin{aligned}
& K_{2}^{n}=-K_{1}^{n}\left(\frac{2 \mathrm{G}_{f}^{N}}{\mathrm{G}_{f}^{N}-\mathrm{G}_{c}^{N}}\right) \frac{b_{N}^{2 n}}{b_{N}^{2 n} \frac{\mathrm{G}_{c}^{N}+\mathrm{G}_{f}^{N}}{\mathrm{G}_{f}^{N}-\mathrm{G}_{c}^{N}}+a_{N}^{2 n}}, \\
& K_{3}^{n}=-K_{1}^{n}\left(\frac{\mathrm{G}_{c}^{N}+\mathrm{G}_{f}^{N}}{\mathrm{G}_{f}^{N}-\mathrm{G}_{c}^{N}}\right) \frac{b_{N}^{2 n}}{b_{N}^{2 n} \frac{\mathrm{G}_{c}^{N}+\mathrm{G}_{f}^{N}}{\mathrm{G}_{f}^{N}-\mathrm{G}_{c}^{N}}+a_{N}^{2 n}}, \\
& K_{4}^{n}=-K_{1}^{n} \frac{a_{N}^{2 n} b_{N}^{2 n}}{b_{N}^{2 n} \frac{\mathrm{G}_{c}^{N}+\mathrm{G}_{f}^{N}}{\mathrm{G}_{f}^{N}-\mathrm{G}_{c}^{N}}+a_{N}^{2 n}} .
\end{aligned}
$$

The stationary values are given by

$$
\beta_{n}=\left(\mathrm{G}_{c}^{N}\right)^{-1}\left(\frac{\mathrm{G}_{f}^{N}\left(b_{N}^{2 n}-a_{N}^{2 n}\right)+\mathrm{G}_{c}^{N}\left(b_{N}^{2 n}+a_{N}^{2 n}\right)}{\mathrm{G}_{f}^{N}\left(b_{N}^{2 n}+a_{N}^{2 n}\right)+\mathrm{G}_{c}^{N}\left(b_{N}^{2 n}-a_{N}^{2 n}\right)}\right) .
$$

One readily checks that for $\mathrm{G}_{f}^{N} \geq \mathrm{G}_{c}^{N}$ that $\beta_{n}$ is increasing with $n$ and that $\beta_{1}=$ $1 / G_{C C A}^{N}$. It can also be easily checked that (5.32) gives all of the stationary values. Indeed one supposes there exists a stationary value $\tilde{\beta}$ not given by (5.32) to find that the only associated stationary functions are of the form $r=$ const., $h=-$ const. Thus we find that $\hat{\beta}=\mathrm{G}_{m} \beta_{1}=\mathrm{G}_{m} / G_{C C A}^{N}$ to conclude that

$$
\text { If } \frac{\mathrm{G}_{m}}{G_{C C A}^{N}} \geq 1 \text {, then } D \leq 0
$$

and Proposition 5.1 follows.

The torsional rigidity for an arbitrary simply connected cross section reinforced with $N$ circular coated fibers is denoted by $\mathcal{T}^{N}(\Omega)$ and repeated application of Proposition 5.1 gives

Proposition 5.2. Upper bound If $\mathrm{G}_{c}^{i} \leq \mathrm{G}_{f}^{i}, i=1, \ldots, N$ and

$$
G_{C C A}^{i} \leq \mathrm{G}_{m}, \text { for } i=1 \ldots, N
$$

then

$$
\mathcal{T}^{N}(\Omega) \leq \mathrm{G}_{m} \mathcal{T}_{0}(\Omega)+\sum_{i=1}^{N}\left(T_{f}^{i}-\frac{\pi}{2} \mathrm{G}_{m} b_{i}^{4}\right),
$$

where $\mathcal{T}_{0}(\Omega)$ is the torsional rigidity of the homogeneous cross section containing material with unit shear modulus. 
Proposition 2.6 follows directly from Proposition 5.2. To establish Proposition 2.4 one recalls the isoperimetric inequality

$$
\mathcal{T}_{0}(\Omega) \leq \frac{\pi}{2} R^{4},
$$

that holds for all cross sections $\Omega$ with area $\pi R^{2}$, see Polya (1948). Proposition 2.4 then follows from (5.36) and (5.35) when $\mathrm{G}_{f}^{i}=\mathrm{G}_{f}$ and $\mathrm{G}_{c}^{i}=\mathrm{G}_{c}$, and $a_{i} / b_{i}=\nu^{1 / 2}$ for $i=1, \ldots, N$.

6. Lower bounds on the torsional rigidity for $\mathrm{G}_{f}^{i} \leq \mathrm{G}_{c}^{i}$. We focus on the case where $\mathrm{G}_{f}^{i} \leq \mathrm{G}_{c}^{i}, i=1, \ldots, N$. We proceed as in the last section and investigate the effects of adding a circular coated fiber to an already existing configuration of $N-1$ coated fibers. At present no assumptions on the geometry or shear moduli of the $N-1$ coated fibers are made. The part of the shaft cross-section already occupied by the coated fibers is denoted by $A$ and the cross-section of the circular coated fiber to be added by $\Sigma$. Here $\Sigma$ is composed of a circular fiber of radius $a_{N}$ with shear modulus $\mathrm{G}_{f}^{N}$ surrounded by a coating of outer radius $b_{N}$ with shear modulus $\mathrm{G}_{c}^{N}$. The torsional rigidity of the original configuration is denoted by $\mathcal{T}(A, \Omega)$. The rigidity associated with the added fiber is written as $\mathcal{T}(A \cup \Sigma, \Omega)$. We state the following:

PROPOSITION 6.1. Lower rigidity inequality

If $\mathrm{G}_{f}^{N} \leq \mathrm{G}_{c}^{N}$ and if

$$
\mathrm{G}_{m} \leq G_{C C A}^{N}
$$

then

$$
\mathcal{T}(A, \Omega)+T_{f}^{N}-\frac{\pi}{2} \mathrm{G}_{m} b_{N}^{4} \leq \mathcal{T}(A \cup \Sigma, \Omega)
$$

Proposition 6.1 is established with the aid of the variational principle given by (2.1). One writes $(2.1)$ as $\mathcal{T}(A \cup \Sigma, \Omega)=-2 \mathcal{E}(A \cup \Sigma, \Omega)$ where

$$
\mathcal{E}(A \cup \Sigma, \Omega)=\min _{\varphi}\left\{\frac{1}{2} \int_{\Omega} \mathcal{G}^{-1}(\mathbf{x})|\nabla \varphi|^{2} d \mathbf{x}-2 \int_{\Omega} \varphi d \mathbf{x}\right\},
$$

where the piecewise constant shear modulus $\mathcal{G}(\mathbf{x})$ is $\mathrm{G}_{m}$ in the matrix and takes the values $\mathrm{G}_{f}^{i}$ and $\mathrm{G}_{c}^{i}$ in the $i^{t h}$ fiber and coating respectively for $i=1, \ldots, N$. The idea of the proof is to estimate the quantity $\mathcal{E}(A \cup \Sigma, \Omega)$ in terms of $\mathcal{E}(A, \Omega)$ associated with the original configuration of $N-1$ fibers. We let $G(\mathbf{x})$ denote the piecewise constant shear modulus for the original configuration $A$ of $N-1$ fibers. Here $G(\mathbf{x})=\mathcal{G}(\mathbf{x})$ outside of $\Sigma$ and inside $\Sigma$ the shear modulus $G(\mathbf{x})=\mathrm{G}_{m}$. We regroup terms in the variational principle (6.3) and write:

$$
\begin{aligned}
\mathcal{E}(A \cup \Sigma, \Omega) & =\min _{\varphi}\left\{\frac{1}{2} \int_{\Omega} G^{-1}(\mathbf{x})|\nabla \varphi|^{2} d \mathbf{x}-2 \int_{\Omega} \varphi d \mathbf{x}+\right. \\
& +\frac{1}{2}\left(\int_{\Sigma}\left(\mathcal{G}^{-1}(\mathbf{x})-\mathrm{G}_{m}^{-1}\right)|\nabla \varphi|^{2} d \mathbf{x}\right\} .
\end{aligned}
$$

We obtain an estimate by substitution of a suitable trial field in (6.4). Our choice is made as follows. We introduce the stress potential $\Phi_{A}$ for the configuration $A$. Here $\Phi_{A}$ is continuous in $\Omega$,

$$
-G^{-1}(\mathbf{x}) \Delta \Phi_{A}=2
$$


and satisfies the transmission conditions

$\mathrm{G}_{f}^{i-1} \nabla \tilde{\Phi}_{\mid f} \cdot \mathbf{n}=\mathrm{G}_{c}^{i-1} \nabla \tilde{\Phi}_{\mid c} \cdot \mathbf{n}$ on the fiber-coating interface, $\left|\mathbf{x}-\mathbf{x}_{i}\right|=a_{i}, i=1, \ldots, N-1$, (6.6)

and

$\mathrm{G}_{f}^{i-1} \nabla \tilde{\Phi}_{\left.\right|_{c}} \cdot \mathbf{n}=\mathrm{G}_{m}{ }^{-1} \nabla \tilde{\Phi}_{\left.\right|_{m}} \cdot \mathbf{n}$ on the matrix-coating interface, $\left|\mathbf{x}-\mathbf{x}_{i}\right|=b_{i}, \ldots, N-1$. (6.7)

The trial field $\varphi$ is chosen to match $\Phi_{A}$ outside the coated fiber cross-section $\Sigma$ but inside we suppose that $\varphi=\Phi_{A}+\delta$. Where $\delta$ is continuous, vanishes on the boundary of $\Sigma$, is square integrable inside $\Sigma$ and has square integrable gradient over $\Sigma$. One easily checks that

$$
\int_{\Sigma} \mathrm{G}_{m}{ }^{-1} \nabla \Phi_{A} \cdot \nabla \delta d \mathbf{x}=2 \int_{\Sigma} \delta d \mathbf{x}
$$

Application of (6.8) and rearranging terms as in the previous section yields:

$$
\begin{aligned}
\mathcal{T}(A \cup \Sigma, \Omega) & \geq \mathcal{T}(A, \Omega)-\int_{\Sigma} \mathrm{G}_{m}{ }^{-1}|\nabla \delta|^{2} d \mathbf{x}+ \\
& +\int_{\Sigma}\left(\mathrm{G}_{m}^{-1}-\mathcal{G}^{-1}(\mathbf{x})\right)|\nabla \varphi|^{2} d \mathbf{x} .
\end{aligned}
$$

On maximizing the right-hand side of (6.9) with respect to $\delta$ we obtain

$$
\mathcal{T}(A \cup \Sigma, \Omega) \geq \mathcal{T}(A, \Omega)+\mathcal{U} .
$$

where

$$
\mathcal{U}=\int_{\Sigma}\left(\mathrm{G}_{m}^{-1}-\mathcal{G}^{-1}(\mathbf{x})\right) \nabla \hat{\varphi} \cdot \nabla \Phi_{A} d \mathbf{x} .
$$

Here $\hat{\varphi}=\hat{\delta}+\Phi_{A}$ in $\Sigma$ and $\hat{\delta}$ solves

$$
\int_{\Sigma} \mathcal{G}^{-1}(\mathbf{x}) \nabla \hat{\delta} \cdot \nabla u d \mathbf{x}=\int_{\Sigma}\left(\mathrm{G}_{m}^{-1}-\mathcal{G}^{-1}(\mathbf{x})\right) \nabla \Phi_{A} \cdot \nabla u d \mathbf{x}
$$

for every trial $u$ vanishing on the boundary of $\Sigma$. From (6.8) and (6.12) we see that $\hat{\varphi}$ solves

$$
\int_{\Sigma} \mathcal{G}^{-1}(\mathbf{x}) \nabla \hat{\varphi} \cdot \nabla u d \mathbf{x}=2 \int_{\Sigma} u d \mathbf{x}
$$

for every trial $u$ vanishing on the boundary of $\Sigma$. Proceeding as in the last section we introduce the continuous functions $\psi, r, \psi^{h}$, and $h$ such that $\hat{\varphi}=\psi-h$ and $\Phi_{A}=r+\psi^{h}$. Here $\psi^{h}$ and $\psi$ are the same functions introduced in Section 5. The function $\psi^{h}$ is the solution of (5.18) and $\psi$ solves the transmission problem given by (5.19) and (5.20). The function $r$ is harmonic in $\Sigma$ and $r=\Phi_{A}$ on the boundary of $\Sigma$. The function $h$ is continuous on $\Sigma$ and $h=-\Phi_{A}$ on the boundary of $\Sigma$. It is the solution of the transmission problem given by (5.21) and (5.22).

Substitution of the functions $\psi^{h}, \psi, r$, and $h$ into $\mathcal{U}$ gives

$$
\mathcal{T}(A \cup \Sigma, \Omega) \geq \mathcal{T}(A, \Omega)+T_{f}^{N}-\frac{\pi}{2} \mathrm{G}_{m} b_{N}^{4}+D,
$$


where the indefinite quantity $D$ is given by (5.25). Its clear that (6.2) holds when $D \geq 0$. We find conditions for which $D \geq 0$ when $r \neq$ const. and $h \neq$ const. To do this we search for the largest number $\rho$ for which

$$
\int_{\Sigma}|\nabla r|^{2} d \mathbf{x}-\rho \int_{\Sigma} \frac{\mathrm{G}_{m}}{\mathcal{G}(\mathbf{x})}|\nabla h|^{2} d \mathbf{x} \geq 0
$$

for every choice of $r$ and $h$ in $\mathcal{C}$ and $r \neq$ const. and $h \neq-$ const. The largest $\rho$ is given by

$$
\hat{\rho}=\inf _{\mathcal{C}} \frac{\int_{\Sigma}|\nabla r|^{2} d \mathbf{x}}{\int_{\Sigma} \frac{\mathrm{G}_{m}}{\mathcal{G}(\mathbf{x})}|\nabla h|^{2} d \mathbf{x}}
$$

Proceeding as in the previous section we find that $\hat{\rho}=G_{C C A}^{N} / \mathrm{G}_{m}$. Thus $D \geq 0$ when $G_{C C A}^{N} / \mathrm{G}_{m} \geq 1$ and the proposition follows.

The torsional rigidity for an arbitrary simply connected cross section reinforced with $N$ circular coated fibers is denoted by $\mathcal{T}^{N}(\Omega)$ and repeated application of Proposition 6.1 gives

PROPOSITION 6.2. Lower bound If $\mathrm{G}_{f}^{i} \leq \mathrm{G}_{c}^{i}, i=1, \ldots, N$ and

$$
\mathrm{G}_{m} \leq G_{C C A}^{i}, \text { for } i=1 \ldots, N
$$

then

$$
\mathrm{G}_{m} \mathcal{T}_{0}(\Omega)+\sum_{i=1}^{N}\left(T_{f}^{i}-\frac{\pi}{2} \mathrm{G}_{m} b_{i}^{4}\right) \leq \mathcal{T}^{N}(\Omega) .
$$

Proposition 2.7 follows directly from Proposition 6.2.

7. Acknowledgments. The work of R.L. was supported by the Air Force Office of Scientific Research, under grant number F49620-02-1-0041 and by the NSF through grant DMS-0296064. The work of T.C. was supported by the National Science Council, Taiwan, under contract NSC 90-2211-E006-119.

\section{REFERENCES}

[1] A. Alvino and G. Trombetti, Isoperimetric inequalities associated with torsion problems and capacity, Bollettino Un. Mat. Ital.,B(6),4(1985),pp. 773-787.

[2] T. Chen, Y. Benveniste, And P. C. Chunng, Exact solution in torsion of composite bars: thickly coated neutral inhomogeneities and composite cylinder assemblages, Proc. R. Soc. Lond.,A458(2002),pp. 1719-1759.

[3] Z. Hashin And B. W. Rosen, The elastic moduli of fiber-reinforced materials, J. Appl. Mech.,31(1964),pp. 222-232.

[4] J. P. Jones And J. S. Whittier, Waves at a flexibly bonded interface, J. Appl. Mech.,34(1967),pp. 905-909.

[5] R. Lipton, Optimal fiber configurations for maximum torsional rigidity, Arch. Rational Mech. Anal.,144(1998),pp. 79-106.

[6] G. W. Milton, The Theory of Composites, Cambridge University Press, Cambridge, UK, 2002.

[7] G. Polya, Torsional rigidity, principle frequency, electrostatic capacity, and symmetrization, Quarterly of Applied Mathematics,6(1948),pp. 267-277.

[8] G. Polya, G. And A. Weinstein, On the torsional rigidity of multiply connected cross sections, Annals of Mathematics,62(1950),pp. 154-163.

[9] G. Polya and G. Szego, Isoperimetric Inequalities in Mathematical Physics, Princeton University Press, Princeton, NJ, 1951.

[10] B, Saint-Venant, Mémoire sur la torsion des prismes, Mém. pres. divers savants Acad. Sci.,14(1856),pp. 233-560. 